\title{
Fast-rotating giant stars behind the Coma Berenices star cluster
}

\author{
Estefanía Casal, ${ }^{\star}$ Matilde Fernández, ${ }^{\star}$ Emilio J. Alfaro, Víctor Casanova and Ángel Tobaruela \\ Instituto de Astrofísica de Andalucía-CSIC, Glorieta de la Astronomía s.n., E-18008 Granada, Spain
}

Accepted 2020 July 9. Received 2020 July 7; in original form 2020 May 16

\begin{abstract}
In the frame of a study of the empirical isochrones of young stellar clusters, we have carried out $B V I_{\mathrm{c}}$ Johnson-Cousins photometry of a sample of $\mathrm{K}$ and $\mathrm{M}$ stars of the Coma Berenices star cluster. All these stars have known rotational periods. Our main goal is to get a valuable reference on the colour-magnitude diagram, $M_{v}$ versus $B-V$, for stars with ages within 400-800 Myr. For this purpose, we obtained $B V I_{\mathrm{c}}$ photometry with an average upper limit for the precision of about $0.025 \mathrm{mag}$ and used parallaxes from the Gaia Data Release 2. We found that one-third of our sample is located well above the cluster main sequence and these stars are confirmed as background giants by their radial velocities in the Gaia Data Release 2. This misclassification shows that giants with short-surface rotational periods can mimic main-sequence stars if they are located at the appropriate distance. We recommend caution when using rotational periods in order to determine cluster membership. Besides, the gyrochronology technique should be used only when the luminosity class of the stars is well known. Finally, our cleared sample supports an age of $\sim 600$ Myr for Coma Berenices, rather than an age of $\sim 800 \mathrm{Myr}$.
\end{abstract}

Key words: Stars: low-mass -Stars: rotation-Galaxy: open clusters and associations: Coma Berenices.

\section{INTRODUCTION}

The Coma Berenices open cluster (Melotte 111, hereinafter Coma Ber) is the second nearest star cluster to the Sun at a distance of $86.7 \mathrm{pc}$ with a dispersion of $7.1 \mathrm{pc}$ (Tang et al. 2018, hereinafter T18). Centred at $\alpha_{J 2000}=12^{h} 25^{m}$ and $\delta_{J 2000}=26^{\circ} 06$ arcmin (Dias et al. 2014), it is very close to the North Galactic Pole. The age of Coma Ber was formerly considered to be between $400 \mathrm{Myr}$ and $600 \mathrm{Myr}$ (e.g. Loktin, Gerasimenko \& Malysheva 2001; Kharchenko et al. 2005; Silaj \& Landstreet 2014). Using gyrochronology, Collier Cameron et al. (2009) obtained an age of $590.7 \pm 40.9$ Myr but recently, T18 estimated an age of around $800 \mathrm{Myr}$ for the cluster through isochrone fitting. In this article, we discuss the age determination of Coma Ber through both techniques.

Isochrone fitting is the most popular method for estimating the age of clusters and stellar associations. The physics behind this technique was well summarized by Angus et al. (2019): 'surface gravity changes resulting from fusion in the core are compared with a set of models that trace stellar evolution across the Hertzsprung-Russell or colour-magnitude diagrams' (HRD and CMD, respectively). A coeval population of stars located at same distance and with similar chemical composition should lie on the same curve (isochrone) in the HRD/CMD. A disadvantage encountered in isochrone fitting, as mentioned by Angus et al. (2019), is the difficulty of obtaining the age for GKM dwarfs, since most of their physical and observable properties do not change rapidly. For these spectral types, gyrochronology might be a better choice to determine their ages.

Empirical age relations are also used to measure ages of stars in open clusters (see review Soderblom 2010). The most commonly used age indicators are lithium depletion (e.g. Sestito \& Randich

^E-mail: ecasal@iaa.es(EC); matilde@iaa.es (MF)
2005), chromospheric activity decay (e.g. Pace \& Pasquini 2004; Pace et al. 2009), and rotational spin-down (gyrochronology).

Gyrochronology, consolidated as a technique by Barnes (2003) and Barnes (2007), is used to derive stellar ages from the rotational periods of the stars and does not depend on stellar distances. Several studies developed in the 1960s suggested a relationship between both rotation and activity with age. Skumanich (1972) plotted projected equatorial rotation velocities $(v \sin i)$ for $\mathrm{G}$ stars belonging to the Pleiades and Hyades open clusters and also for the Sun. He showed that rotation decayed as the inverse square root of age. Subsequently, Kawaler (1989) suggested the possibility of determining the age of a late-type star from its rotation and colour.

Barnes (2003) and Barnes (2007) selected data of stars in open clusters and the Sun to calibrate the relationship between rotational period, colour, and age. In this way, gyrochronology moved from $v \sin i$ to rotational periods, getting rid of the extra uncertainty given by $v \sin i$, due to the poor knowledge of the angle between the rotation axis and the line of sight (i). Barnes (2003) and Barnes (2007) plotted period versus $B-V$ for stars of young clusters and older field stars and observed that coeval stars were placed in two different sequences that he defined as slow/interface/I and fast/convective/C. The I sequence contained the stars with longer rotational periods, which followed the Skumanich relationship between rotation and age. Moreover, he characterized each coeval population with a common rotation-age relation that resulted from the fit to the I sequence. Subsequent works performed new calibrations in order to get new empirical rotation-age relations for the I sequence (e.g. Mamajek \& Hillenbrand 2008; Meibom, Mathieu \& Stassun 2009). In the last decades, rotational periods for solar and late-type stars have been measured in Coma Ber (Radick, Skiff \& Lockwood 1990; Marilli, Catalano \& Frasca 1997; Collier Cameron et al. 2009; Terrien et al. 2014) and used to estimate the age of the cluster by using gyrochronology (Collier Cameron et al. 2009; Angus et al. 2015). 
Coma Ber has a large sky coverage $\left(>13^{\circ} \times 8^{\circ}\right)$ and for this reason, membership studies struggle with discriminating between members and field stars, as it is clear in the thorough study carried out by Sampedro et al. (2017) on a very large sample of clusters. The first membership study of Coma Ber was performed by Trumpler (1938), who analysed proper motions, photometry, radial velocities (RVs), and spectral types, and identified 37 bright members and seven fainter probable members. The next notorious effort to increase the number of members (Casewell, Jameson \& Dobbie 2006), made use of proper motions and photometry, confirmed 45 previously known members and identified 60 new late-type candidate members. Subsequent works, Kraus \& Hillenbrand (2007), Mermilliod, Grenon \& Mayor (2008), Collier Cameron et al. (2009), Melnikov \& Eislöffel (2012), and Terrien et al. (2014), used photometry and several of the kinematic parameters (positions, proper motions, and RVs) and identified a total of $\sim 150$ candidate members for Coma Ber before the era of Gaia.

Gaia provided an improvement on the accuracy of fundamental parameters used in membership studies: Gaia Data Release 1 (Gaia DR1, Gaia Collaboration 2016) and Gaia Data Release 2 (Gaia DR2, Gaia Collaboration 2018a), which led to more reliable results on the candidate members list of Coma Ber. Gaia Collaboration (2017) employed positions, proper motions, and parallax data from Gaia DR1 to analyse a field of $10.4^{\circ}$ around the Coma Ber centre and reported 50 possible members. T18, collecting proper motions, photometry data, and distance measurements from parallaxes by Gaia DR2, provided a list with 192 stellar and substellar member candidates. Within that list, 148 stars were expected to be true members. Moreover, T18 not only undertook their own membership study but also collected and checked the results of previous membership studies for Coma Ber, except for the membership study by Collier Cameron et al. (2009). Tang et al. (2019) (hereinafter T19) explained that the previous study of T18 might have included field stars in the candidate members list, due to a large radius cut of $17 \mathrm{mas} / \mathrm{yr}$ on the proper motions selection. Furthermore, T19 identified leading and trailing tails of Coma Ber and provided a list of 197 Coma Ber candidate members by making use of Gaia DR2 astrometry.

In the frame of our study of the isochrone fitting technique for GKM stars of young/intermediate-age open clusters (30-800 Myr), we compiled photometry for late-type (late $\mathrm{G}$ to $\mathrm{M}$ dwarfs) candidate members of the Coma Ber cluster with available rotational periods. Stellar distances were taken from Bailer-Jones et al. (2018), which were estimated using Gaia DR2 data and Bayesian inference (hereinafter BJ distances). These BJ distances reveal that several stars had been wrongly classified as $\mathrm{K}$ dwarfs members of Coma Ber. In this article, we will discuss the nature of these stars and analyse the consequences of this misclassification for the technique of gyrochronology as an age determination tool.

\section{OBSERVATIONS}

\subsection{The sample}

We selected young low-mass stars (late $\mathrm{G}$ to $\mathrm{M}$ dwarfs) that had been catalogued as Coma Ber candidate members by one or several of the following membership studies: Casewell et al. (2006), Kraus \& Hillenbrand (2007), Collier Cameron et al. (2009), and Terrien et al. (2014). Among them we chose those stars with measured rotational periods in the literature. The selection process was undertaken before 2017. Hence, we did not take into account the membership studies of Gaia Collaboration (2017), Sampedro et al. (2017), T18, and T19.
Our sample contains 34 stars (G8 to M0), 33 of them have rotational periods measured by Collier Cameron et al. (2009), who identified 37 variable stars as late $\mathrm{F}$ to $\mathrm{M}$ candidate members of Coma Ber with membership probabilities higher than 50 per cent. Among them, 20 stars were regarded as probable new members. They obtained rotational periods for these variable stars measuring the period of their variability through observations by SuperWASP. The rotational period of the remaining star in our sample was determined by Marilli et al. (1997). Three of the stars with periods obtained by Collier Cameron et al. (2009) were also measured by Terrien et al. (2014). For two of these stars, we calculated the average of the periods obtained by both studies. For the third star, we kept the period of Collier Cameron et al. (2009), since the one from Terrien et al. (2014) diverged from the expected value for a late-type star.

We were able to obtain $B V I_{\mathrm{c}}$ photometry for 33 out of 34 stars and for the remaining star, we took $B, V$ magnitudes from Kharchenko (2001). In order to include additional photometric information to our sample, we also collected photometry from $J H K_{\mathrm{s}} 2 \mathrm{MASS}$ (Skrutskie et al. 2006), Pan-STARRS DR1 (Chambers et al. 2016), UKIDSSDR9 (Lawrence et al. 2013), and SDSS-III DR12 (Alam et al. 2015). Moreover, we gathered parallaxes, proper motions, and RVs from Gaia DR2 and, as we mentioned before, BJ distances.

Table A1 shows the membership, photometry, parallaxes, proper motions, RVs, and rotational periods of the selected sample. The calibrated magnitude $M_{v}$ was derived taking into account the BJ distances, also shown in the table. In order to compare these distances with the ones determined by the inverse of Gaia DR2 parallaxes, we present the results corresponding to both methods and as expected, the lower the parallaxes, the greater the discrepancies.

\subsection{Photometry}

Calibrated photometry has been carried out from observations made with the 1.5-m telescope at Observatorio de Sierra Nevada (OSN), Spain, during nine nights (from 2015 May to 2017 May). We used $B V I_{\mathrm{c}}$ Johnson-Cousins filters and, attached to the telescope, a VersArray $2048 \times 2048$ CCD with a pixel size of $13.5 \mu \mathrm{m}$ and a field of view of $7.92 \times 7.92$ arcmin.

Our Coma Ber sample contains stars with $B-V$ values from 0.65 to $1.55 \mathrm{mag}$. In order to perform the absolute calibration, we carefully selected a sample of standard stars from Landolt (1992), Landolt (2009), and Landolt (2013), and located in the following standard star fields: SA32, SA98, SA101, SA104, and SA109. These standard stars covered the $B-V$ values range from 0.5 to 2.2 mag.

The CCD images were bias and flat-field corrected to remove instrumental signatures across the detector. Each night was processed independently with the IRAF software (Image Reduction and Analysis Facility). We conducted aperture photometry and, in order to obtain the calibrated magnitudes, we applied the transformation equations developed by Harris, Fitzgerald \& Reed (1981) to the instrumental magnitudes for the required $B V I_{\mathrm{c}}$ Johnson-Cousins filters.

With the objective to estimate the error of our transformation from the instrumental to the calibrated system, we have computed the internal errors as defined by Harris et al. (1981). Internal errors (in mag) range from 0.007 to 0.022 for the $B-V$ index, from 0.008 to 0.022 for $V$, and from 0.009 to 0.020 for the $V-I$ index. Average values of the extinction coefficients for $B-V, V$, and $V-I$ are $0.060,0.158$, and $0.116 \mathrm{mag} / \mathrm{airmass}$. For the $V$ band, we got values as large as 0.22 and $0.26 \mathrm{mag} /$ airmass, which we attribute to dust in the atmosphere, and we also got three very transparent nights with extinction below $0.13 \mathrm{mag} /$ airmass (the OSN lies at $2896 \mathrm{~m}$ of altitude). 


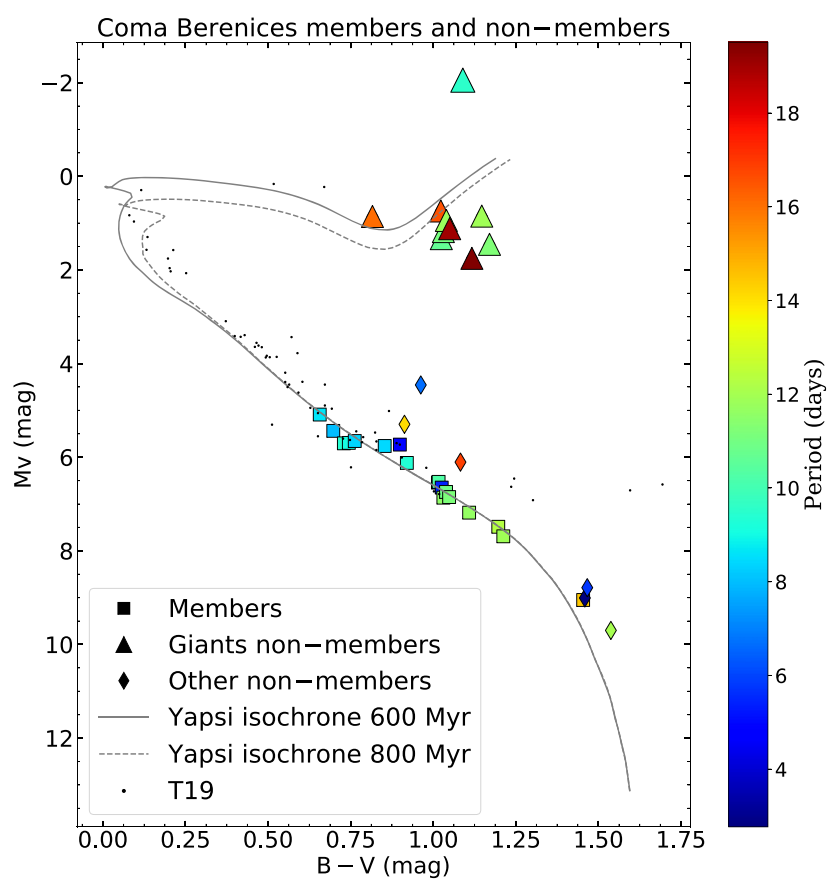

Figure 1. CMD for our sample of Coma Ber stars with known periods. We also show the candidate members from T19, represented by dots, with $B V$ photometry from Kharchenko (2001). The isochrones correspond to Yapsi evolutionary tracks of $600 \mathrm{Myr}$ and $800 \mathrm{Myr}$ (solid and dashed lines, respectively). Ten stars out of 34 are located well above the main sequence, one of them is situated over the giant branch, the remaining nine lie close to this branch. Several stars almost overlap and might be difficult to distinguish in the CMD and their periods and photometry can be checked in Table A1.

For each star and filter, we have computed an individual error that is actually an upper limit of the real error. We obtained these individual errors as the square root of the squared sum of the upper limit residual and of the error of the instrumental magnitudes of the stars. The upper limit residual was the highest value among the photometric residuals for the selected standard stars. The mean value of the upper limit errors of the sample for the $V$ magnitude and the $B$ $-V, V-I_{\mathrm{c}}$ colours is $\sim 0.025 \mathrm{mag}$.

Results are listed in Table A1. For the target Cl* Melotte 111 AV 1431, the photometry was taken from Kharchenko (2001). The uncertainty of its colour and magnitude, $B-V$ and $V$, is $\sim 0.3 \mathrm{mag}$ and $\sim 0.1$ mag, respectively.

\section{RESULTS}

\subsection{Coma Ber membership in our sample}

Fig. 1 shows the position of the stars of our sample (mainly K dwarfs) in the CMD. For completeness sake, we included stars with spectral types earlier than $\mathrm{K}$ by plotting all the candidate members from T19 with available $B V$ photometry from Kharchenko (2001). We overplotted two isochrones that belong to the set of Yale-Potsdam Stellar Isochrones (YaPSI, Spada et al. 2017) and correspond to solar metallicity and ages of $600 \mathrm{Myr}$ and $800 \mathrm{Myr}$.

Ten stars, with $M_{v}<2 \mathrm{mag}$, are located well above the cluster main sequence in the $\mathrm{CMD}$. Nine of them show BJ distances between $\sim 950 \mathrm{pc}$ and $\sim 1550 \mathrm{pc}$ or, in terms of Gaia DR2 parallaxes, between $\sim 0.6$ mas and $\sim 1$ mas (the mean Gaia DR2 parallax of Coma Ber is $\sim 11.6$ mas). Considering their calibrated magnitude $M_{v}$, with values from $\sim 0.7$ to $\sim 1.8 \mathrm{mag}$, and their colour index $B-V$, from $\sim 0.8$ to $\sim 1.2 \mathrm{mag}$, we concluded that they might be non-member giant stars (luminosity class III). Unfortunately, neither $V-I$ nor the nearinfrared colours help discriminating $\mathrm{K}$ giants from $\mathrm{K}$ dwarfs ( $\mathrm{Li}$ et al. 2016). Nevertheless, the giant assumption is supported by their RVs, since two differentiated groups are presented in the Gaia DR2 RVs of our sample. The RVs in the first group, including only the Coma Ber main-sequence stars, range from $\sim-6$ to $\sim 3 \mathrm{~km} / \mathrm{s}$. The second group, which contains only the giant stars, has the following RVs range: $\sim-70<\mathrm{RV}<\sim-18 \mathrm{~km} / \mathrm{s}$ and $\sim 12<\mathrm{RV}<\sim 164 \mathrm{~km} / \mathrm{s}$.

We observe a star with $M_{v} \sim-2 \mathrm{mag}$ in our CMD, 2MASSJ12394200 +2134578 , which is located at a BJ distance of $\sim 5270 \mathrm{pc}$ and has a Gaia DR2 parallax value of $\sim 0.09$ mas. This star is even brighter than the nine stars classified as class III and belongs to the luminosity class II of bright giants. Its RV, 164.51 $\mathrm{km} / \mathrm{s}$, is in agreement with the RV expected for halo stars (Harris \& Racine 1979).

After discarding these 10 giants, 24 stars remained in our sample. As some of them might have also been misclassified as Coma Ber members, we checked T18 and T19 studies. Both utilized data from Gaia DR2, which provided higher accuracy than the catalogues of Gaia DR1 and UCAC4 (The fourth United States Naval Observatory CCD Astrograph Catalog, Zacharias et al. 2013) used by Gaia Collaboration (2017) and Sampedro et al. (2017), respectively. Hence, we only took into account the membership information from T18 and T19. A total of 17 stars were identified as candidate members by one of the studies or by both of them. Since T19 reported that their previous work (T18) might have included field stars in their candidate members list, we analysed the proper motions of the six stars that were considered candidate members by T18 but not by T19. We would keep classifying as candidate members those with proper motions $(\mu)$ similar to the mean values for Coma Ber given by Gaia Collaboration (2018b): $\mu_{\alpha}=-12.111 \pm 0.0048 \mathrm{mas} / \mathrm{yr}$ and $\mu_{\delta}=$ $-8.996 \pm 0.121$ mas/yr. Three stars satisfied this criterion, while the other three had proper motions that slightly diverged from the mean values of the cluster, with a maximum difference of \pm 4 mas/yr. We checked the position of those stars in the CMD and corresponded to that of the candidate members. Therefore, we kept all the 17 stars as candidate members of Coma Ber.

We still had seven stars from our sample of 24 that did not have membership information in any of the studies: T18 and T19. In order to avoid a possible misclassification, we checked their BJ distances and Gaia DR2 proper motions. We marked out as probable nonmembers the stars with a distance higher than $94 \mathrm{pc}$, since Coma Ber has an average measured distance of $86.7 \pm 7.1 \mathrm{pc}$ (T18). For the stars with BJ distances smaller than 94 pc, we analysed their proper motions, as described above. We finally classified six out of the seven stars as probable non-members of Coma Ber; four of them were rejected by the distance criterion and the other two by analysing the proper motions.

In summary, we identified 16 stars as non-members of the cluster, of which 10 are giant stars and six are other probable non-members.

Our sample was then originally contaminated because the work by Collier Cameron et al. (2009), although thorough, adopted a wide interval of proper motions. Their candidate members had the following ranges of Gaia DR2 proper motions: $\sim-21<\mu_{\alpha}<\sim-5$ mas/yr, $\sim 0<\mu_{\alpha}<\sim 21 \mathrm{mas} / \mathrm{yr}$, and $\sim-17<\mu_{\delta}<\sim-4$ mas/yr, $\sim 0<\mu_{\delta}<\sim 8$ mas/yr.

The proper motions of all the stars in our sample are represented in Fig. 2, where we see how candidate members are preferentially concentrated in a region while non-members are more scattered. 


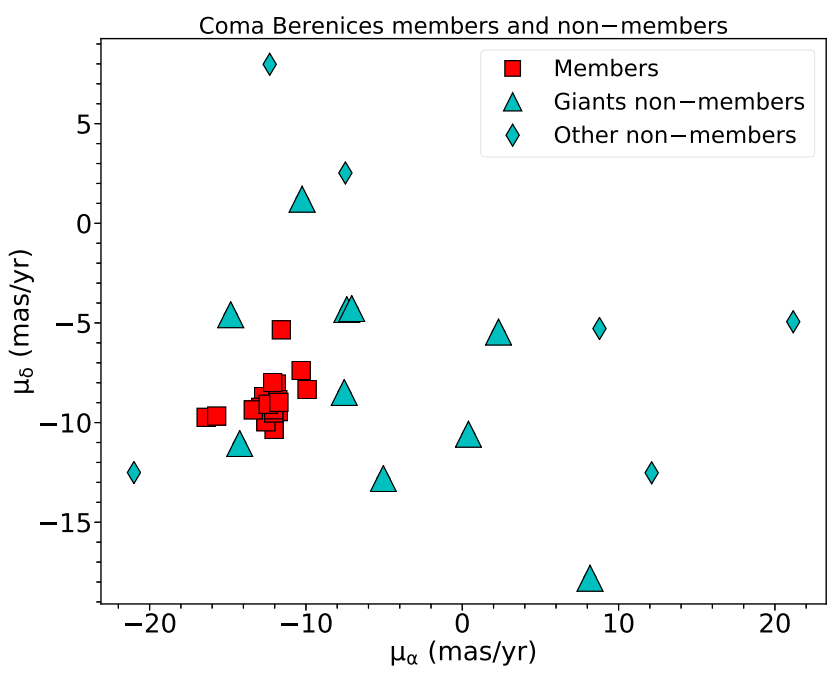

Figure 2. Proper motions of Coma Ber candidate members and nonmembers. Most of the candidate members are concentrated in a differentiated region of proper motions with respect to the stars that do not belong to the cluster.

\subsection{Rotational periods of the giant stars in our sample}

In this section, we intend to understand why our sample has been contaminated with giant background stars.

If we compare the surface rotational periods (from 9.77 to $19.51 \mathrm{~d}$ ) of our subsample of giant stars with those of the giants observed by Kepler and analysed by Ceillier et al. (2017), we notice that our subsample matches the scarcely populated group of fast rotators, as defined by them.

We want to compute what fraction of the giant population represents the fast rotators, with period below $23 \mathrm{~d},{ }^{1}$ both for the Kepler giants and for our subsample. The fraction of fast rotators in the Kepler sample can be calculated with the information given by Ceillier et al. (2017), which studied red giants (luminosity class III). In order to compute the fraction for our subsample, we have to estimate the size of the whole sample to which our giants belong. Using the Gaia DR2 search tool, we looked for all the giant stars that share the spatial, photometric, and dynamical space with our nine giants of luminosity class III. Therefore, we selected the stars that are in the ranges covered by the nine giants: $G$ band means magnitude between 11.20 and $12.10 \mathrm{mag} ; B P-R P$ colour index between 1.045 mag and 1.351 mag; $\alpha, \delta$ coordinates: $\alpha$ from $11^{h} 48^{m}$ to $13^{h} 05^{m}$, and $\delta$ from $19^{\circ}$ to $43^{\circ}$; and proper motions constrained to the limits used by Collier Cameron et al. (2009) and parallaxes between 0.365 mas and 1.153 mas. This range of parallaxes corresponds to distances from 867 to $2740 \mathrm{pc}$, which were calculated taking into account the difference in magnitude between early $\mathrm{K}$ dwarfs and giants in the CMD: K giants are on average 100-1000 times more luminous and hence can 'simulate' main-sequence dwarfs if they are located 10-32 times further away. We found 628 giants that fulfil all the mentioned requirements.

The nine giants of our subsample represent 1.4 per cent of these 628 giants. This percentage is of the same order than the 0.6 per cent fraction of low-mass red giants that exhibit surface rotational periods with values below $23 \mathrm{~d}$ in the Kepler sample analysed by Ceillier et al.

${ }^{1}$ This upper limit comes from the bins that Ceillier et al. (2017) used for their fig.5.

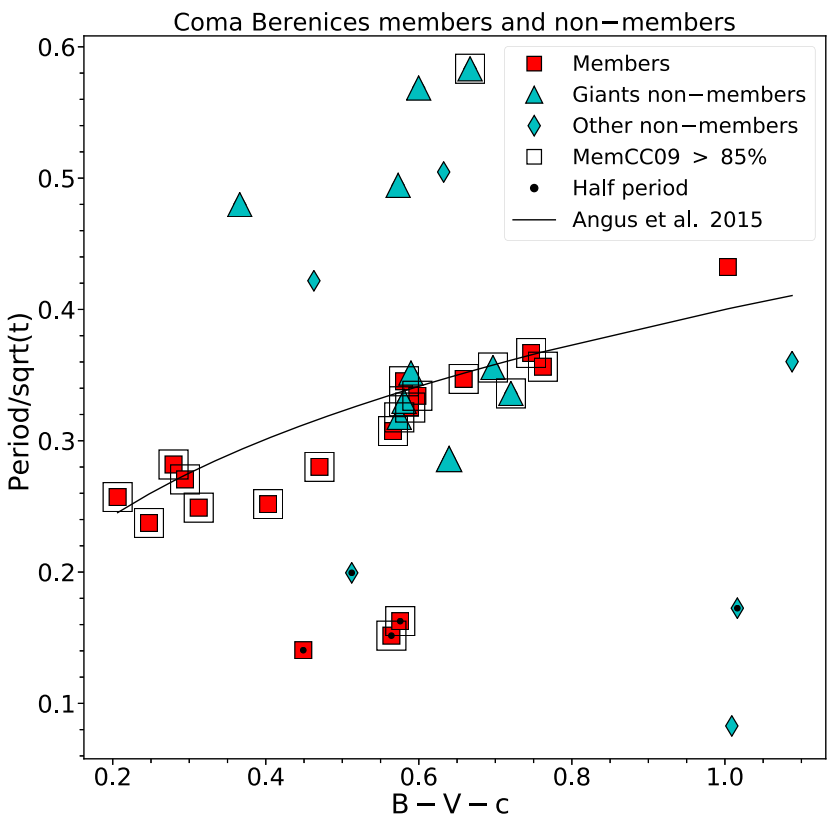

Figure 3. Period-colour relation for our Coma Ber sample. In red, we represent the stars we considered members of the cluster and, in cyan, the stars we marked out as non-members. Giant stars are shown by triangles. Inside the hollow square symbol, we show the stars in our sample that were used by Collier Cameron et al. (2009) for their period-colour calibration. Symbols containing a black dot represent the stars with periods considered double their published values by Collier Cameron et al. (2009). The solid line represents the fit of the period-colour relation (equation 1), being $c=0.45$, from Angus et al. (2015) for an age of $t=590 \mathrm{Myr}$.

(2017). Therefore, we think we have included in our sample most of the giants that could be mistaken as Coma Ber members because they mimic the Coma Ber main-sequence K stars.

Carlberg et al. (2011) compiled three mechanisms that might explain the high rotational velocities of some giant stars: interactions from a close binary companion; core-envelope differential rotation, where the core rotates much faster than the surface; and a planet spiralling towards the star that inserts angular momentum into the convective envelope of the star.

The Kepler field points through the Galaxy disc and our study has been done along a direction perpendicular to the disc. Interestingly, there seems to be a similar fraction of very fast-rotating giants in both directions.

\section{DISCUSSION}

\subsection{Gyrochronology}

Collier Cameron et al. (2009) followed the same method described in Barnes (2007), who considered that the period is inversely proportional to the square root of the age, and determined a periodcolour relation for the Coma Ber cluster. They used the periods from Radick et al. (1990) (not included in our study) and Marilli et al. (1997), their periods of stars with membership probabilities higher than 85 per cent, and the 2MASS $J-K$ colours. Using the aforementioned period-colour relation, Collier Cameron et al. (2009) obtained an empirical calibration of period as a function of age and colour and inferred an age of 590.7 \pm 40.9 Myr for Coma Ber.

In Fig. 3, we plotted period versus $B-V$ for our sample of stars, highlighting those that were used for the period-colour calibration by 
Collier Cameron et al. (2009). We also marked out the stars for which Collier Cameron et al. (2009) used the double of the published period: these are stars for which they analysed their periods and concluded that the true periods were twice their estimations.

Two groups of giant non-members are shown in Fig. 3. The first one contains six out of the 10 giants with period values from 9.6 to $11.9 \mathrm{~d}$, which are the fastest rotators among the giants. They fit well within the range of periods of the main-sequence stars (between 9.4 and $14.5 \mathrm{~d}$ ). This first group mimics the Coma Ber main-sequence stars in colour, period, and apparent magnitude. The second group contains the four remaining giants, which are slower rotators (periods from 16.0 to $19.5 \mathrm{~d}$ ), and does not follow the trend line of the main-sequence members. Both groups might affect the calibration of gyrochronology: the fast rotators would add noise into the fitting of the main sequence, and the slower rotators might be more problematic, since they can modify the fitting for the calibration in a higher level than the first group.

Regarding the calibration of gyrochronology by Collier Cameron et al. (2009), they made a cut in their candidate members list, and, as we mentioned before, discarded the periods of stars with membership probabilities lower than 85 per cent. As a consequence, only five giants contaminated their selected sample for the period-colour calibration. Four giants mimic well the main-sequence members. The remaining giant appears at the top of the plot. They considered the possibility that this star was not a member and decided to not take it into account for the calibration. For those reasons, the giants in the study by Collier Cameron et al. (2009) barely modified the fit to the main-sequence stars for their calibration of gyrochronology.

Angus et al. (2015), on the other side, used the whole sample of Coma Ber stars selected by Collier Cameron et al. (2009) in order to calibrate their gyrochronology relation:

$P=A^{0.55} \times 0.40(B-V-0.45)^{0.31}$,

where $P$ is the rotational period (days) and $A$ the age (Myr). They included both the main-sequence and the giant stars, because the giants had not been identified as such. Their figs 1 and 2 show for Coma Ber a tail of slower rotators that are slower than the Hyades, although they consider Coma Ber (500 Myr) younger than the Hyades (625 Myr). The three slowest rotators are in fact background giant stars that add noise to the calibration. Fig. 3 shows their periodcolour relation that corresponds to equation (1), for an age of $590 \mathrm{Myr}$, and the result seems to indicate an agreement with the age of $590.7 \pm 40.9$ Myr given by Collier Cameron et al. (2009). Nevertheless, once the giants had been removed, the best fitting to our data would be a slightly younger age.

In future studies of stellar ages using the gyrochronology technique, one should be aware of this problematic contamination of giants that do not belong to the cluster under study.

\subsection{Age of Coma Berenices}

There is a disagreement between the $\sim 800$-Myr isochronal age obtained by T18 and the gyrochronological age of $590.7 \pm 40.9 \mathrm{Myr}$ obtained by Collier Cameron et al. (2009).

The isochronal age determination by T18 relied on five evolved stars in the cluster region. One of these five stars $(18 \mathrm{Com})$ was excluded by $\mathrm{T} 18$, based on its distance and proper motions. Two evolved stars are binary systems, and another star $(16 \mathrm{Com})$ lied on both the 600-Myr and 800-Myr isochrones. Only one star (14 Com) seems to be an isolated star that supports the $800-$ Myr estimation. Nevertheless, T19 discarded 14 Com as a candidate member of the Coma Ber cluster.
Our study eliminates the slowest rotators of the rotational periods distribution in the Coma Ber cluster, because they have been identified as background giants. In this way, the Hyades and the Coma Ber period distributions share the shape of the slow rotators tail. Therefore, the age of both clusters should be quite similar.

For all these reasons, we are more confident with the age determination obtained from the gyrochronology technique that gives an age of $590.7 \pm 40.9 \mathrm{Myr}$ (Collier Cameron et al. 2009).

\section{CONCLUSIONS}

We study the position on the CMD for late-type stars located in the Coma Ber cluster. We obtained $B V I_{\mathrm{c}}$ photometric data from observations and compiled rotational periods from the literature. These are the main results of this work:

(i) Distances obtained from Gaia DR2 parallaxes have shown nine stars located in the red-giant branch on the CMD and one bright giant, bringing to light the existence of giants previously identified as $\mathrm{K}$ dwarfs in our sample. The search of stellar cluster members based on rotational periods needs to take into account the possible contamination of background red giants by checking their individual parallaxes.

(ii) Rotational periods of these giant stars show that they are fast rotators, and their values do not correspond to the common periods related to their spectral type and luminosity class. Six out of the 10 giants have periods that mimic well with the periods of the mainsequence stars. The remaining four stars are slower rotators than the other six giants and do not follow the trend of the main-sequence stars.

(iii) The nine $\mathrm{K}$ giants of our sample represent, among the giants that share spatial, photometric, and dynamical properties with them, a small percentage (1.4 per cent) as the giants with surface rotational periods below $23 \mathrm{~d}$ ( 0.6 per cent) in the whole sample of 17377 oscillating red giants of Ceillier et al. (2017). A similar percentage has also been found by other surveys of giant stars.

(iv) Contamination of giant stars might be a problem in the process of determining stellar ages from the gyrochronology technique, because the giant stars can add noise to the rotational period distribution. Therefore, before using the gyrochronology technique, it is necessary to confirm the luminosity class of the stars.

(v) In the frame of the discrepancy between the ages determined by isochrones and gyrochronology, T19 have discarded as a Coma Ber candidate member, the evolved star that was crucial in T18 for their age estimation of $\sim 800 \mathrm{Myr}$ by using the isochrone fitting technique. Besides, after removing the background giants, the rotational period distribution of Coma Ber fits better the age estimated by the gyrochronology technique. Therefore, we are more confident with the result obtained by gyrochonology, which gives an age of $590.7 \pm 40.9 \mathrm{Myr}$ (Collier Cameron et al. 2009) for the Coma Ber cluster.

\section{ACKNOWLEDGEMENTS}

This research was supported by the Spanish AYA2016-79425-C3-3$\mathrm{P}$ grant of the Spanish Ministry of Economy and Competitiveness (MINECO), co-funded with EU FEDER funds. EC acknowledges financial support from the Spanish Ministry of Economy and Competitiveness under the FPI grant AYA2011-30147-C03-01. EJA acknowledges financial support from the State Agency for Research of the Spanish MCIU through grant PGC2018-095049-B- 
C21. AT acknowledges financial support from the Spanish National Research Council (CSIC) through the Intramural grant 201850E126. We also acknowledge support from the State Agency for Research of the Spanish MCIU through the 'Center of Excellence Severo Ochoa' award for the Instituto de Astrofísica de Andalucía (SEV2017-0709). This research was based on data taken at the Sierra Nevada Observatory, which is operated by the Instituto de Astrofisica de Andalucia (CSIC). This work has made use of data from the European Space Agency (ESA) mission Gaia (https://www.cosmos .esa.int/gaia), processed by the Gaia Data Processing and Analysis Consortium (DPAC, https://www.cosmos.esa.int/web/gaia/dpac/con sortium). Funding for the DPAC has been provided by national institutions, in particular, the institutions participating in the Gaia Multilateral Agreement. This research has made use of the SIMBAD database, the VizieR catalogue access tool, and 'Aladin sky atlas', CDS, Strasbourg, France; of TOPCAT (Taylor 2005); of PYTHON (http://www.python.org), NUMPY (van der Walt, Colbert \& Varoquaux 2011), Pandas (Mckinney 2011), and MATPLOTLIB (Hunter 2007); and of IRAF, distributed by the National Optical Astronomy Observatory, which is operated by the Association of Universities for Research in Astronomy (AURA) under a cooperative agreement with the National Science Foundation. We thank the anonymous referee for reading the paper carefully and providing thoughtful comments, many of which have resulted in changes in the paper.

\section{DATA AVAILABILITY}

The authors confirm that the data supporting the findings of this study are available within the article.

\section{REFERENCES}

Alam S. et al., 2015, ApJS, 219, 12

Angus R. et al., 2019, AJ, 158, 173

Angus R., Aigrain S., Foreman-Mackey D., McQuillan A., 2015, MNRAS, 450, 1787

Bailer-Jones C. A. L., Rybizki J., Fouesneau M., Mantelet G., Andrae R., 2018, AJ, 156, 58

Barnes S. A., 2003, ApJ, 586, 464

Barnes S. A., 2007, ApJ, 669, 1167

Carlberg J. K., Majewski S. R., Patterson R. J., Bizyaev D., Smith V. V., Cunha K., 2011, ApJ, 732, 39

Casewell S. L., Jameson R. F., Dobbie P. D., 2006, MNRAS, 365, 447

Ceillier T. et al., 2017, A\&A, 605, A111

Chambers K. C. et al., 2016, preprint(arXiv:1612.05560)

Collier Cameron A. et al., 2009, MNRAS, 400, 451

Dias W. S., Monteiro H., Caetano T. C., Lépine J. R. D., Assafin M., Oliveira A. F., 2014, A\&A, 564, A79
Gaia Collaboration, 2016, A\&A, 595, A2

Gaia Collaboration, 2017, A\&A, 601, A19

Gaia Collaboration, 2018a, A\&A, 616, A1

Gaia Collaboration, 2018b, A\&A, 616, A10

Harris W. E., Racine R., 1979, ARA\&A, 17, 241

Harris W. E., Fitzgerald M. P., Reed B. C., 1981, PASP, 93, 507

Hunter J. D., 2007, Comput. Sci. Eng., 9, 90

Kawaler S. D., 1989, ApJ, 343, L65

Kharchenko N. V., 2001, Kinematika Fiz. Nebesnykh Tel, 17, 409

Kharchenko N. V., Piskunov A. E., Röser S., Schilbach E., Scholz R. D., 2005, A\&A, 438, 1163

Kraus A. L., Hillenbrand L. A., 2007, AJ, 134, 2340

Landolt A. U., 1992, AJ, 104, 340

Landolt A. U., 2009, AJ, 137, 4186

Landolt A. U., 2013, AJ, 146, 131

Lawrence A. et al., 2013, VizieR Online Data Catalog. p. II/319

Li J. et al., 2016, ApJ, 823, 59

Loktin A. V., Gerasimenko T. P., Malysheva L. K., 2001, Astron. Astrophys. Trans., 20, 607

Mamajek E. E., Hillenbrand L. A., 2008, ApJ, 687, 1264

Marilli E., Catalano S., Frasca A., 1997, Mem. Soc. Astron. Ital., 68, 895

Mckinney W., 2011, Python High Performance Science Computer

Meibom S., Mathieu R. D., Stassun K. G., 2009, ApJ, 695, 679

Melnikov S., Eislöffel J., 2012, A\&A, 544, A111

Mermilliod J. C., Grenon M., Mayor M., 2008, A\&A, 491, 951

Pace G., Pasquini L., 2004, A\&A, 426, 1021

Pace G., Melendez J., Pasquini L., Carraro G., Danziger J., François P., Matteucci F., Santos N. C., 2009, A\&A, 499, L9

Radick R. R., Skiff B. A., Lockwood G. W., 1990, ApJ, 353, 524

Sampedro L., Dias W. S., Alfaro E. J., Monteiro H., Molino A., 2017, MNRAS, 470, 3937

Sestito P., Randich S., 2005, A\&A, 442, 615

Silaj J., Landstreet J. D., 2014, A\&A, 566, A132

Skrutskie M. F. et al., 2006, AJ, 131, 1163

Skumanich A., 1972, ApJ, 171, 565

Soderblom D. R., 2010, ARA\&A, 48, 581

Spada F., Demarque P., Kim Y. C., Boyajian T. S., Brewer J. M., 2017, ApJ, 838,161

Tang S.-Y. et al., 2019, ApJ, 877, 12

Tang S.-Y., Chen W. P., Chiang P. S., Jose J., Herczeg G. J., Goldman B., 2018, ApJ, 862, 106

Taylor M. B., 2005, TOPCAT \&amp; STIL: Starlink Table/VOTable Processing Software. p. 29

Terrien R. C. et al., 2014, ApJ, 782, 61

Trumpler R. J., 1938, Lick Obs. Bull., 494, 167

van der Walt S., Colbert S. C., Varoquaux G., 2011, Comput. Sci. Eng., 13, 22

Zacharias N., Finch C. T., Girard T. M., Henden A., Bartlett J. L., Monet D. G., Zacharias M. I., 2013, AJ, 145, 44 


\section{APPENDIX A: ADDITIONAL TABLE}

Table A1. Coma Ber members and non-members with measured periods.

\begin{tabular}{|c|c|c|c|c|c|c|c|c|c|c|c|c|c|c|c|c|c|c|}
\hline$\overline{\text { Name }}$ & $\begin{array}{l}\alpha_{J 2000} \\
(\mathrm{~h}: \mathrm{m}: \mathrm{s})\end{array}$ & $\begin{array}{l}\delta_{22000} \\
\left({ }^{\circ} !: "\right.\end{array}$ & $\begin{array}{c}\begin{array}{c}\text { Memb. } \\
\text { notes }\end{array} \\
\end{array}$ & $\begin{array}{l}B-V \\
(\mathrm{mag})\end{array}$ & $\begin{array}{c}\mathrm{e} B-V \\
(\mathrm{mag})\end{array}$ & $\begin{array}{c}V \\
(\mathrm{mag})\end{array}$ & $\begin{array}{c}\mathrm{eV} \\
(\mathrm{mag})\end{array}$ & $\begin{array}{c}M_{v} \\
(\mathrm{mag})\end{array}$ & $\begin{array}{l}V-I \\
(\mathrm{mag})\end{array}$ & $\begin{array}{c}\mathrm{e} V-I \\
(\mathrm{mag})\end{array}$ & $\begin{array}{c}\text { Plx } \\
\text { (mas) }\end{array}$ & $\begin{array}{c}D_{\text {inv.Gaia }} \\
(\mathrm{pc})\end{array}$ & $\begin{array}{c}D_{\text {BJ.Gaia }} \\
(\mathrm{pc})\end{array}$ & $\begin{array}{c}\mu_{\alpha} \\
(\mathrm{mas} / \mathrm{yr})\end{array}$ & $\begin{array}{c}\mu_{\delta} \\
(\mathrm{mas} / \mathrm{yr})\end{array}$ & $\begin{array}{c}R V \\
(\mathrm{Km} / \mathrm{s})\end{array}$ & $\begin{array}{l}\text { Period } \\
\text { (days) }\end{array}$ & Flag \\
\hline 2 2MASS J11483770 + 2816305 & $11: 48: 37.70$ & $+28: 16: 30.5$ & & 0.75 & 0.03 & 10.39 & 0.03 & 5.70 & 0.78 & 0.03 & 11.20 & 89.29 & 89.06 & -12.06 & -9.36 & 0.50 & 9.43 & $\mathrm{~b}$ \\
\hline 2MASS J11553336 + 2943417 & $11: 55: 33.35$ & $+29: 43: 41.7$ & & 1.03 & 0.04 & 11.64 & 0.03 & 6.87 & 1.16 & 0.03 & 11.06 & 90.45 & 90.22 & -15.71 & -9.67 & 2.93 & 11.55 & $\mathrm{~b}$ \\
\hline 2MASS J12005224 + 2719237 & $12: 00: 52.24$ & $+27: 19: 23.9$ & NM & 1.02 & 0.03 & 11.50 & 0.03 & 1.33 & 1.09 & 0.03 & 0.89 & 1120.65 & 1082.84 & -10.24 & 1.21 & 30.84 & 10.63 & b \\
\hline 2MASS J12022298 + 2254590 & $12: 02: 22.86$ & $+22: 54: 58.7$ & NM & 0.91 & 0.02 & 11.72 & 0.02 & 5.30 & 0.92 & 0.02 & 5.17 & 193.54 & 192.48 & -7.48 & 2.53 & & 14.11 & b \\
\hline $\mathrm{Cl} *$ Melotte $111 \mathrm{AV} 189$ & 12:07:57.72 & $+25: 35: 11.3$ & & 1.02 & 0.02 & 11.36 & 0.02 & 6.53 & 1.09 & 0.02 & 10.78 & 92.73 & 92.50 & -9.93 & -8.34 & -6.08 & 10.28 & b \\
\hline 2MASS J12083610 + 3106098 & 12:08:36.10 & $+31: 06: 09.9$ & & 1.21 & 0.02 & 12.43 & 0.03 & 7.69 & 1.43 & 0.01 & 11.24 & 88.98 & 88.76 & -11.89 & -8.07 & -0.54 & 11.92 & $\mathrm{~b}$ \\
\hline 2MASS J12113516 + 2922444 & 12:11:35.11 & $+29: 22: 44.5$ & & 1.01 & 0.02 & 11.29 & 0.02 & 6.54 & 1.03 & 0.02 & 11.19 & 89.33 & 89.11 & -11.80 & -8.84 & 0.91 & $(5.07)$ & $\mathrm{b}$ \\
\hline $\mathrm{Cl} *$ Melotte 111 AV 523 & $12: 12: 53.23$ & $+26: 15: 01.3$ & & 1.03 & 0.02 & 11.32 & 0.03 & 6.65 & 1.07 & 0.02 & 11.59 & 86.29 & 86.08 & -12.07 & -9.52 & 0.59 & (5.44) & $\mathrm{b}$ \\
\hline $\mathrm{Cl} *$ Melotte $111 \mathrm{AV} 1183$ & $12: 21: 15.62$ & $+26: 09: 14.0$ & & 1.04 & 0.02 & 11.38 & 0.03 & 6.74 & 1.13 & 0.02 & 11.78 & 84.92 & 84.71 & -11.76 & -9.45 & -0.28 & 10.88 & $\mathrm{~b} *$ \\
\hline Cl* Melotte 111 AV 1404 & $12: 23: 47.22$ & $+23: 14: 44.3$ & & 1.05 & 0.02 & 11.50 & 0.03 & 6.85 & 1.16 & 0.02 & 11.75 & 85.10 & 84.89 & -12.56 & -9.96 & 0.55 & 11.18 & $\mathrm{~b}$ \\
\hline $\mathrm{Cl} *$ Melotte $111 \mathrm{AV} 1431$ & $12: 24: 06.00$ & $+26: 07: 43.0$ & & 0.90 & 0.30 & 10.47 & 0.12 & 5.73 & & & 11.25 & 88.90 & 88.67 & -11.73 & -8.99 & -0.04 & $(4.7)$ & $\mathrm{a}, \mathrm{c}$ \\
\hline 2MASS J12260132 + 3421084 & $12: 26: 01.31$ & $+34: 21: 08.4$ & NM & 1.15 & 0.02 & 11.64 & 0.02 & 0.85 & 0.02 & 1.12 & 0.66 & 1509.69 & 1440.89 & 0.39 & -10.58 & -37.06 & 11.90 & $\mathrm{~b}$ \\
\hline $\mathrm{Cl} *$ Melotte $111 \mathrm{AV} 1660$ & $12: 26: 51.03$ & $+26: 16: 01.8$ & & 1.11 & 0.02 & 11.85 & 0.03 & 7.18 & 1.26 & 0.02 & 11.63 & 85.97 & 85.76 & 12.13 & -7.98 & 0.70 & 11.61 & d \\
\hline 2MASS J12265646 + 2240542 & $12: 26: 56.48$ & $+22: 40: 54.7$ & NM & 1.47 & 0.02 & 15.11 & 0.03 & 8.79 & 1.82 & 0.02 & 5.42 & 184.63 & 183.66 & 8.78 & -5.28 & & $(5.77)$ & $\mathrm{b}$ \\
\hline Cl* Melotte 111 AV 1693 & $12: 27: 20.68$ & $+23: 19: 47.4$ & & 0.74 & 0.03 & 10.31 & 0.03 & 5.70 & 0.82 & 0.03 & 11.90 & 84.05 & 83.85 & -12.41 & -9.08 & 0.89 & 9.05 & $\mathrm{~b}$ \\
\hline Cl* Melotte 111 AV 1737 & $12: 27: 48.29$ & $+28: 11: 39.8$ & & 0.66 & 0.03 & 9.65 & 0.03 & 5.09 & 0.70 & 0.03 & 12.23 & 81.78 & 81.59 & -13.37 & -9.36 & -0.08 & 8.60 & $\mathrm{~b}$ \\
\hline $\mathrm{Cl} *$ Melotte $111 \mathrm{AV} 1826$ & $12: 28: 56.43$ & $+26: 32: 57.4$ & & 0.92 & 0.03 & 10.75 & 0.02 & 6.12 & 0.95 & 0.02 & 11.86 & 84.35 & 84.15 & -12.88 & -9.24 & 1.30 & 9.37 & d \\
\hline Cl* Melotte 111 AV 1889 & $12: 29: 42.15$ & $+28: 37: 14.6$ & NM & 0.82 & 0.03 & 11.28 & 0.02 & 0.86 & 0.90 & 0.02 & 0.79 & 1266.52 & 1216.11 & -7.55 & -8.49 & 61.33 & 16.05 & $\mathrm{~b}$ \\
\hline 2MASS J12332002 + 2224234 & $12: 33: 20.01$ & $+22: 24: 23.3$ & & 0.76 & 0.03 & 10.27 & 0.03 & 5.65 & 0.82 & 0.03 & 11.92 & 83.93 & 83.73 & -10.30 & -7.38 & -1.81 & 8.33 & $\mathrm{~b}$ \\
\hline 2MASS J12334186 + 2914016 & $12: 33: 41.88$ & $+29: 14: 01.7$ & NM & 1.08 & 0.04 & 11.48 & 0.03 & 6.11 & 1.19 & 0.03 & 8.37 & 119.47 & 119.07 & 21.17 & -4.94 & -8.03 & 16.88 & b \\
\hline $\mathrm{Cl} *$ Melotte $111 \mathrm{AV} 2177$ & $12: 33: 42.12$ & $+25: 56: 34.0$ & & 0.85 & 0.02 & 10.53 & 0.02 & 5.76 & 0.88 & 0.02 & 11.09 & 90.15 & 89.920 & -16.38 & -9.74 & -1.37 & 8.42 & $\mathrm{~b}$ \\
\hline $\mathrm{Cl} *$ Melotte $111 \mathrm{MGM} 427$ & $12: 33: 54.22$ & $+27: 08: 04.7$ & NM & 1.02 & 0.02 & 11.58 & 0.03 & 0.74 & 0.99 & 0.02 & 0.65 & 1547.39 & 1469.82 & -14.81 & -4.59 & 12.81 & 16.54 & $\mathrm{~b}$ \\
\hline 2 MASS J12381145 + 2333223 & $12: 38: 11.47$ & $+23: 33: 22.2$ & & 1.45 & 0.01 & 13.75 & 0.01 & 9.05 & 1.82 & 0.03 & 11.45 & 87.32 & 87.10 & -12.04 & -10.34 & -0.84 & 14.46 & $\mathrm{~b}$ \\
\hline 2MASS J12394200 + 2134578 & $12: 39: 41.99$ & $+21: 34: 58.0$ & NM & 1.09 & 0.04 & 11.58 & 0.03 & -2.05 & 1.24 & 0.03 & 0.09 & 11587.57 & 5266.94 & -7.07 & -4.28 & 164.51 & 9.57 & $\mathrm{~b}$ \\
\hline 2 MASS J12423510 + 4105276 & $12: 42: 35.14$ & $+41: 05: 27.7$ & NM & 1.04 & 0.02 & 11.83 & 0.01 & 0.94 & 1.07 & 0.02 & 0.63 & 1576.48 & 1503.11 & -7.39 & -4.33 & 15.86 & 11.76 & $\mathrm{~b}$ \\
\hline 2MASS J12430953 + 2447052 & $12: 43: 09.53$ & $+24: 47: 05.2$ & NM & 1.46 & 0.02 & 13.88 & 0.03 & 9.01 & 2.06 & 0.02 & 10.55 & 94.78 & 94.54 & 12.12 & -12.52 & -1.07 & 2.77 & b \\
\hline 2MASS J12452334 + 4251041 & $12: 45: 23.35$ & $+42: 51: 04.4$ & NM & 1.54 & 0.02 & 14.38 & 0.03 & 9.70 & 2.43 & 0.02 & 11.56 & 86.49 & 86.28 & -21.00 & -12.51 & & 12.05 & $\mathrm{~b}$ \\
\hline 2MASS J12490041 + 2521356 & 12:49:00.42 & $+25: 21: 35.6$ & & 1.20 & 0.02 & 12.03 & 0.02 & 7.49 & 1.37 & 0.02 & 12.29 & 81.34 & 81.16 & -12.71 & -8.69 & & 12.27 & $\mathrm{~b}$ \\
\hline 2MASS J12493043 + 2532109 & $12: 49: 30.43$ & $+25: 32: 11.1$ & NM & 1.12 & 0.02 & 11.65 & 0.02 & 1.75 & 1.14 & 0.02 & 1.02 & 979.84 & 952.50 & -14.24 & -11.05 & -21.31 & 19.51 & b \\
\hline 2MASS J12500170 + 2103121 & 12:50:01.70 & $+21: 03: 12.1$ & NM & 0.96 & 0.02 & 11.25 & 0.02 & 4.46 & 0.98 & 0.02 & 4.42 & 226.30 & 228.48 & -12.32 & 7.98 & -24.86 & $(6.67)$ & $\mathrm{b}$ \\
\hline 2MASS J12531465 + 2403135 & $12: 53: 14.67$ & $+24: 03: 13.6$ & NM & 1.05 & 0.04 & 12.09 & 0.03 & 1.12 & 1.16 & 0.03 & 0.62 & 1625.96 & 1546.16 & 8.17 & -17.81 & -70.74 & 19.02 & $\mathrm{~b}$ \\
\hline 2MASS J12573686 + 2858448 & $12: 57: 36.86$ & $+28: 58: 44.7$ & & 0.72 & 0.03 & 10.24 & 0.03 & 5.44 & 0.80 & 0.03 & 10.90 & 91.74 & 91.50 & -11.57 & -5.34 & -0.92 & 7.94 & $\mathrm{~b}$ \\
\hline 2MASS J12592775 + 1941150 & $12: 59: 27.75$ & $+19: 41: 15.1$ & NM & 1.17 & 0.02 & 11.83 & 0.02 & 1.45 & 1.17 & 0.02 & 0.81 & 1231.36 & 1186.91 & -5.05 & -12.81 & -59.56 & 11.24 & $\mathrm{~b}$ \\
\hline 2MASS J13054399 + 2003214 & 13:05:43.99 & $+20: 03: 21.4$ & NM & 1.03 & 0.02 & 11.20 & 0.03 & 1.18 & 1.03 & 0.02 & 0.96 & 1041.05 & 1010.34 & 2.32 & -5.46 & -18.84 & 11.04 & b \\
\hline
\end{tabular}

Note. Parameters shown in this $13.05 ; 3.99+20.03: 21.4 \quad \mathrm{NM}$ $V$, and $V-I$, except for the star Cl* Melotte $111 \mathrm{AV} 1431$ for which we took photometry from Kharchenko (2001); calibrated magnitude $M_{v}$, obtained using distances by Bailer-Jones et al. (2018); parallaxes by Gaia DR2 (Plx); distances taken from Gaia DR2 parallaxes by the inverse of the parallax values ( $\left.D_{\text {inv.Gaia }}\right)$ and by the Bayesian analysis of Bailer-Jones et al. $(2018)\left(D_{B J . G a i a}\right)$; proper motions and radial velocities taken from Gaia DR2; and periods taken from literature (see Notes). The periods in between '()' were multiplied by 2 by Collier Cameron et al. (2009).

Notes.

${ }^{a}$ Photometry from Kharchenko (2001)

${ }^{b}$ Period from Collier Cameron et al (2009).

${ }_{b *}^{b *}$ Period from Collier Cameron et al. (2009), chosen between the values given by Collier Cameron et al. (2009) and Terrien et al. (2014).

${ }^{c}$ Period from Marilli et al. (1997).

${ }^{d}$ Mean value of the periods from Collier Cameron et al. (2009) and Terrien et al. (2014).

This paper has been typeset from a $\mathrm{T}_{\mathrm{E}} \mathrm{X} / \mathrm{LT} \mathrm{T} \mathrm{X}$ file prepared by the author. 